\title{
AN ELEMENTARY APPROACH TO A LATTICE-VALUED BANACH-STONE THEOREM
}

\section{매III}

\author{
JINXI CHEN
}

\begin{abstract}
Let $X$ and $Y$ be compact Hausdorff spaces, and $E$ be a nonzero real Banach lattice. In this note, we give an elementary proof of a lattice-valued Banach-Stone theorem by Cao, Reilly and Xiong [3] which asserts that if there exists a Riesz isomorphism $\Phi: C(X, E) \rightarrow C(Y, \mathbb{R})$ such that $\Phi(f)$ has no zeros if $f$ has none, then $X$ is homeomorphic to $Y$ and $E$ is Riesz isomorphic to $\mathbb{R}$.
\end{abstract}

\section{Introduction}

Let $X$ and $Y$ be compact Hausdorff spaces, and $E, F$ be nonzero real Banach lattices. Let $C(X, E)$ denote the Banach lattice of all continuous $E$-valued functions on $X$ equipped with the pointwise ordering and the sup norm. Let $\mathbb{R}$ be the Banach lattice of real numbers with the usual norm and order. Note that in general, Riesz isomorphism (i.e., lattice isomorphism) of $C(X, E)$ and $C(X, F)$ does not necessarily imply topological homeomorphism of $X$ and $Y$ (see [3]). Recently, Cao, Reilly and Xiong [3] established the following lattice-valued Banach-Stone theorem:

Theorem A.([3], Theorem 3.3) Suppose there is a Riesz isomorphism $\Phi: C(X, E) \rightarrow$ $C(Y, \mathbb{R})$ such that $\Phi(f)$ has no zeros if $f$ has none. Then $X$ is homeomorphic to $Y$ and $E$ is Riesz isomorphic to $\mathbb{R}$.

Towards their proof of Theorem A, they considered the support for a Riesz homomorphism and gave the following:

Theorem B.([3], Theorem 2.2) If $\Phi: C(X, E) \rightarrow \mathbb{R}$ is a Riesz homomorphism such that $\Phi\left(\mathbf{1}_{X} \otimes e\right) \neq 0$ if $e \neq 0$, then $\Phi$ has a unique support.

In this short note we claim that Theorems A and B mentioned above can be deduced from the following two well-known results, respectively:

Received November 23, 2005.

2000 Mathematics Subject Classification. Primary 46E40; Secondary 46B42, 46E05

Key words and phrases. Banach lattice, Riesz homomorphism, Banach-Stone theorem.

This work is supported in part by the National Natural Science Foundation of China under Grant NO.10571090. 
Theorem $\mathbf{A}^{\prime}$.([8]; cf. [1], Theorem 7.22) If $C(X, \mathbb{R})$ and $C(Y, \mathbb{R})$ are Riesz isomorphic, then $X$ and $Y$ homeomorphic.

Theorem $\mathbf{B}^{\prime}$.([8]; cf. [1], Theorem 7.21) For any Riesz homomorphism $\Phi: C(X, \mathbb{R}) \rightarrow$ $\mathbb{R}$ with $\Phi\left(\mathbf{1}_{X}\right)=1$ there exists a unique $a \in X$ such that $\Phi(f)=f(a)$ for each $f \in C(X, \mathbb{R})$.

Our elementary proof also establishes the conjecture posed by Cao, Reilly and Xiong which asserts that Theorem $\mathrm{A}^{\prime}$ implies Theorem A.

In this note we mostly follow the notion and notations used in [3]. For $\omega \in C(X, \mathbb{R})$ and $e \in E$, let $\omega \otimes e \in C(X, E)$ be defined by $(\omega \otimes e)(x)=\omega(x) e$ for each $x \in X$. We call $a \in X$ a support for a Riesz homomorphism $\Phi: C(X, E) \rightarrow F$ if $\Phi(f)=\Phi\left(\mathbf{1}_{X} \otimes f(a)\right)$ for every $f \in C(X, E)$, where $\mathbf{1}_{X} \in C(X, \mathbb{R})$ is defined by $\mathbf{1}_{X}(x)=1$ for all $x \in X$. If $\Phi: C(X, E) \rightarrow C(Y, \mathbb{R})$ is a Riesz homomorphism, then define $\hat{\Phi}(y)(u)=\Phi\left(\mathbf{1}_{X} \otimes u\right)(y)$ for each $u \in E$ and each $y \in Y$. Clearly, $\hat{\Phi}(y)$ is a linear functional on $E$ for each $y \in Y$. For undefined terms and notions refer to [1] and [3].

\section{The Elementary Proofs of Theorems A and B}

We start with the proof of Theorem B.

Proof of Theorem B. First we claim that the Banach lattice $E$ is Riesz isomorphic to $\mathbb{R}$, in notation $E \cong \mathbb{R}$. Suppose on the contrary that $E$ is not Riesz isomorphic to $\mathbb{R}$. Then there would exist two elements $e_{1}, e_{2} \in E^{+} \backslash\{0\}$ such that $e_{1} \wedge e_{2}=0$ (see [4], P. 19). Since $\left(\mathbf{1}_{X} \otimes e_{1}\right) \wedge\left(\mathbf{1}_{X} \otimes e_{2}\right)=0$ in $C(X, E)$ and $\Phi$ is a Riesz isomorphism, we have $\Phi\left(\mathbf{1}_{X} \otimes e_{1}\right) \wedge \Phi\left(\mathbf{1}_{X} \otimes e_{2}\right)=0$ in $\mathbb{R}$, which implies that $\Phi\left(\mathbf{1}_{X} \otimes e_{1}\right)=0$ or $\Phi\left(\mathbf{1}_{X} \otimes e_{2}\right)=0$. This is impossible. Let $u$ be an arbitrary nonzero element of $E^{+}$. Then $E=\{\lambda u: \lambda \in \mathbb{R}\}$. Next, we note that to every $f \in C(X, E)$ there corresponds a unique $\omega_{f} \in C(X, \mathbb{R})$ such that $f=\omega_{f} \otimes u$. Clearly, this correspondence is a Riesz isomorphism of $C(X, E)$ onto $C(X, \mathbb{R})$.

Now, let $\Gamma(\omega)=\Phi(\omega \otimes u)$ for $\omega \in C(X, \mathbb{R})$. It is obvious that $\Gamma: C(X, \mathbb{R}) \rightarrow \mathbb{R}$ is a Riesz homomorphism and $\Gamma\left(\mathbf{1}_{X}\right)=\Phi\left(\mathbf{1}_{X} \otimes u\right)>0$. By Theorem $\mathrm{B}^{\prime}$, there exists a unique $a \in X$ such that $\Gamma(\omega)=\omega(a) \Phi\left(\mathbf{1}_{X} \otimes u\right)$ for each $\omega \in C(X, \mathbb{R})$. Therefore, for every $f=\omega_{f} \otimes u \in C(X, E)$ we have

$$
\begin{aligned}
\Phi(f)=\Phi\left(\omega_{f} \otimes u\right) & =\Gamma\left(\omega_{f}\right) \\
& =\omega_{f}(a) \Phi\left(\mathbf{1}_{X} \otimes u\right) \\
& =\Phi\left(\mathbf{1}_{X} \otimes\left(\omega_{f}(a) u\right)\right) \\
& =\Phi\left(\mathbf{1}_{X} \otimes f(a)\right) .
\end{aligned}
$$

So $a$ is also a support for $\Phi$.

It remains to show that $\Phi$ has a unique support. Let $a_{1}, a_{2} \in X$ be such that $\Phi(f)=\Phi\left(\mathbf{1}_{X} \otimes f\left(a_{1}\right)\right)=\Phi\left(\mathbf{1}_{X} \otimes f\left(a_{2}\right)\right)$ for all $f \in C(X, E)$. Then we have $f\left(a_{1}\right)=f\left(a_{2}\right)$ for every $f \in C(X, E)$, which implies $a_{1}=a_{2}$. 
The proof of Theorem A. As proved in Lemma 3.1 of $[3], E \cong \mathbb{R}$ (The proof of surjectivity of $\hat{\Phi}(y)$ is superfluous since the range space is $\mathbb{R})$. Therefore it follows from the proof of Theorem $\mathrm{B}$ that $C(X, \mathbb{R}) \cong C(X, E) \cong C(Y, \mathbb{R})$. In view of Theorem $\mathrm{A}^{\prime}$ we can see that $X$ and $Y$ are homeomorphic.

We can say more about the Riesz isomorphism $\Phi$. As done in the proof of Theorem $\mathrm{B}$, let $u \in E^{+} \backslash\{0\}$ be fixed, and let the Riesz isomorphism $\Psi: C(X, \mathbb{R}) \rightarrow C(X, E)$ be defined by $\Psi(\omega)=\omega \otimes u$ for each $\omega \in C(X, \mathbb{R})$. Clearly, $\Phi \circ \Psi$ is a Riesz isomorphism of $C(X, \mathbb{R})$ onto $C(Y, \mathbb{R})$. Then there exists a unique positive function $\pi \in C(Y, \mathbb{R})$ and an (onto) homeomorphism $\phi: Y \rightarrow X$ such that

$$
[(\Phi \circ \Psi)(\omega)](y)=\pi(y) \omega(\phi(y))
$$

for all $y \in Y$ and all $\omega \in C(X, \mathbb{R})$ ( see, e.g. [1], Theorem 7.22). Here $\pi=(\Phi \circ \Psi)\left(\mathbf{1}_{X}\right)$, and $\pi(y)>0$ for every $y \in Y$. Now, for every $f=\omega_{f} \otimes u \in C(X, E)$ and $y \in Y$, we have

$$
[\Phi(f)](y)=\left[(\Phi \circ \Psi)\left(\omega_{f}\right)\right](y)=\pi(y) \omega_{f}(\phi(y))=\Pi(y) f(\phi(y)),
$$

where $\Pi(y)$ is a Riesz isomorphism of $E$ onto $\mathbb{R}$ satisfying $\Pi(y)(\lambda u)=\lambda \pi(y), \lambda \in \mathbb{R}$. That is, $\Phi$ can be written as a weighted composition operator.

Remark 1. Under the hypothesis of Theorem A, $\Phi$ and $\Phi^{-1}$ are disjointness preserving operators. Then the homeomorphism of $Y$ onto $X$ and the representation of $\Phi$ as a weighted composition operator can be obtained from Gau, Jeang and Wong [5, Theorem $2.3]$.

Remark 2. In the above discussion and [3], the compactness of $X$ and $Y$ plays a key role. If we weaken the compactness of $X$ and $Y$ to realcompactness, then the conclusion of Theorem $\mathrm{A}$ is still valid. Indeed, we still have $E \cong \mathbb{R}$ and $C(X, \mathbb{R}) \cong C(X, E) \cong C(Y, \mathbb{R})$ as we did in the above proof of Theorem A. Then the desired conclusion follows from Proposition 3 of [2].

If $\Phi: C(X, E) \rightarrow C(Y, \mathbb{R})$ is a linear bijection such that $\Phi f$ has no zeros if, and only if, $f$ has no zeros, then $X, Y$ are homeomorphic even without the hypothesis that $\Phi$ is a Riesz isomorphism, which is required for Theorem A. To prove this we need the following proposition. Recall that a continuous scalar function is invertible whenever it has no zeros.

Proposition. Let $X, Y$ be compact Hausdorff connected topological spaces. Let $T$ : $C(X, \mathbb{R}) \rightarrow C(Y, \mathbb{R})$ be a linear bijection such that $T f$ is invertible in $C(Y, \mathbb{R})$ if, and only if, $f$ is invertible in $C(X, \mathbb{R})$. Then there is a homeomorphism $\sigma$ from $Y$ onto $X$ and a strictly positive or negative function $h$ in $C(Y, \mathbb{R})$ such that

$$
T f=h \cdot f \circ \sigma, \quad \forall f \in C(X, \mathbb{R}) .
$$


Proof. First note that the invertible function $T \mathbf{1}_{X}$ is either strictly positive or strictly negative on $Y$. Assume $0<m<T \mathbf{1}_{X}(y)<M, \forall y \in Y$. We claim that $\Phi f \geq 0$ whenever $f \geq 0$. Indeed, $T f$ must assume positive values at some points. For else, $T f-\delta T \mathbf{1}_{X}<0$ for all $\delta>0$. Then $f-\delta$ is invertible. But this is impossible for some $\delta$. Suppose $T f$ also assumed negative values. Let $\epsilon>0$ be small enough that $T f+\epsilon T \mathbf{1}_{X}$ still assumes both positive and negative values. In particular, $T f+\epsilon T \mathbf{1}_{X}$ is not invertible. Thus $f+\epsilon$ is not invertible, a contradiction.

Let $x \in X$ and let $M_{x}$ be the subspace of $C(X, \mathbb{R})$ consisting of all functions $f$ in $C(X, \mathbb{R})$ with $f(x)=0$. Let

$$
\operatorname{Ker} T M_{x}=\left\{y \in Y: T f(y)=0, \forall f \in M_{x}\right\}
$$

We claim that $\operatorname{Ker} T M_{x}$ is non-empty. Suppose, on contrary, that $\operatorname{Ker} T M_{x}$ is empty. Then for each $y$ in $Y$ there is an $f_{y}$ in $M_{x}$ such that $T f_{y}$ is nonzero at $y$, and thus in a neighborhood of $y$. We can assume further that both $f_{y}$ and $T f_{y}$ are non-negative, by replacing them by their positive parts or negative parts. By compactness of $Y$, we can choose finitely many positive $f_{1}, \ldots, f_{n}$ from $M_{x}$ such that the positive functions $T f_{1}, \ldots, T f_{n}$ have no common zero in $Y$. Hence $T\left(f_{1}+\cdots+f_{n}\right)$ is strictly positive, and thus invertible. This conflicts with the fact that $f_{1}+\cdots+f_{n}$ vanishes at $x$.

Next, we claim that $\operatorname{Ker} T M_{x}$ is indeed a singleton. Indeed, if $y_{1}, y_{2} \in \operatorname{Ker} T M_{x}$ then we have $T M_{x} \subseteq M_{y_{i}}, \quad i=1,2$. Applying the above argument for $T^{-1}$, we shall have $T^{-1} M_{y_{i}} \subseteq M_{x_{i}}$ for some $x_{i}$ in $X, i=1,2$. However, this gives $T M_{x} \subseteq M_{y_{i}} \subseteq$ $T M_{x_{i}}, \quad i=1,2$. It follows from the bijectivity of $T$ that $x=x_{1}=x_{2}$. Thus,

$$
T M_{x}=M_{y_{1}}=M_{y_{2}} \quad \text { and } \quad y_{1}=y_{2}
$$

We can now define a bijective map $\sigma: Y \rightarrow X$ such that

$$
T M_{\sigma(y)}=M_{y}, \quad \forall y \in Y
$$

Hence there is a (real) scalar function $h$ on $Y$ such that

$$
T f(y)=h(y) f(\sigma(y)), \quad \forall y \in Y \text {. }
$$

Clearly, $h=T \mathbf{1}_{X}$ is a strictly positive function in $C(Y, \mathbb{R})$. It is then routine to see that $\sigma$ is a homeomorphism from $Y$ onto $X$. For the proof refer to [6], [7].

Now let $E$ be a Banach space. Let $\Phi: C(X, E) \rightarrow C(Y, \mathbb{R})$ is a linear bijection such that $\Phi f$ has no zeros if, and only if, $f$ has no zeros. Then, for each $y \in Y, \hat{\Phi}(y)$ defined by $\hat{\Phi}(y)(u)=\Phi\left(\mathbf{1}_{X} \otimes u\right)(y)$ is a linear isomorphism from $E$ onto $\mathbb{R}$. Let $u$ be an arbitrary nonzero element of $E$. Let $\Psi: C(X, \mathbb{R}) \rightarrow C(X, E)$ be defined by $\Psi(\omega)=\omega \otimes u$ for each $\omega \in C(X, \mathbb{R})$. Clearly, $\Phi \circ \Psi$ is a linear isomorphism of $C(X, \mathbb{R})$ onto $C(Y, \mathbb{R})$ such that $(\Phi \circ \Psi) \omega$ is invertible in $C(Y, \mathbb{R})$ if and only if $\omega$ is invertible in $C(X, \mathbb{R})$. Therefore, we have proved the following corollary. 
Corollary. Let $X, Y$ be compact Hausdorff connected topological spaces. If $\Phi$ : $C(X, E) \rightarrow C(Y, \mathbb{R})$ is a linear bijection such that $\Phi f$ has no zeros if, and only if, $f$ has no zeros, then $X, Y$ are homeomorphic.

\section{Acknowledgement}

The author would like to express deep thanks to Professor Ngai-Ching Wong for his helpful remarks, in particular, for the Proposition.

\section{References}

[1] C. D. Aliprantis and O. Burkinshaw, Positive Operators, Academic Press, New York, 1985.

[2] J. Araujo, E. Beckenstein and L. Narici, Biseparating maps and homeomorphic realcompactifications, J. Math. Anal. Appl. 192(1995), 258-265.

[3] J. Cao, I. Reilly and H. Xiong, A lattice-valued Banach-Stone theorem, Acta Math. Hungar., 98 (2003), 103-110.

[4] D. H. Fremlin, Topological Riesz Spaces and Measure Theory, Cambridge Univ. Press, London, 1974.

[5] H.-L. Gau, J.-S. Jeang and N.-C. Wong, Biseparating linear maps between continuous vector-valued function spaces, J. Aust. Math. Soc., 74 (2003), 101-109.

[6] H.-L. Gau, J.-S. Jeang and N.-C. Wong, An algebraic approach to the Banach-Stone theorem for separating linear bijections, Taiwanese J. Math., 6(2002), 399-403.

[7] J.-S. Jeang and N.-C. Wong, On the Banach-Stone Problem, Studia Math., 155 (2003), 95-105.

[8] E. de Jonge and A. van Rooij, Introduction to Riesz Spaces, Mathematical Centre Tracts 78, Amsterdam, 1978.

Department of Mathematics, Southwest Jiaotong University, Chengdu 610031, P.R. China.

E-mail: chenjinxi@mail.nankai.edu.cn 